This item was submitted to Loughborough's Research Repository by the author.

Items in Figshare are protected by copyright, with all rights reserved, unless otherwise indicated.

\title{
Conductive lithographic film fabricated resistive strain gauges
}

PLEASE CITE THE PUBLISHED VERSION

http://dx.doi.org/10.1109/ICSENS.2003.1278937

PUBLISHER

(c) IEEE

VERSION

VoR (Version of Record)

LICENCE

CC BY-NC-ND 4.0

\section{REPOSITORY RECORD}

Hay, Gareth I., Peter S.A. Evans, David J. Harrison, Darren J. Southee, George Simpson, and Paul M. Harrey. 2019. "Conductive Lithographic Film Fabricated Resistive Strain Gauges". figshare.

https://hdl.handle.net/2134/8478. 
This item was submitted to Loughborough's Institutional Repository (https://dspace.lboro.ac.uk/) by the author and is made available under the following Creative Commons Licence conditions.

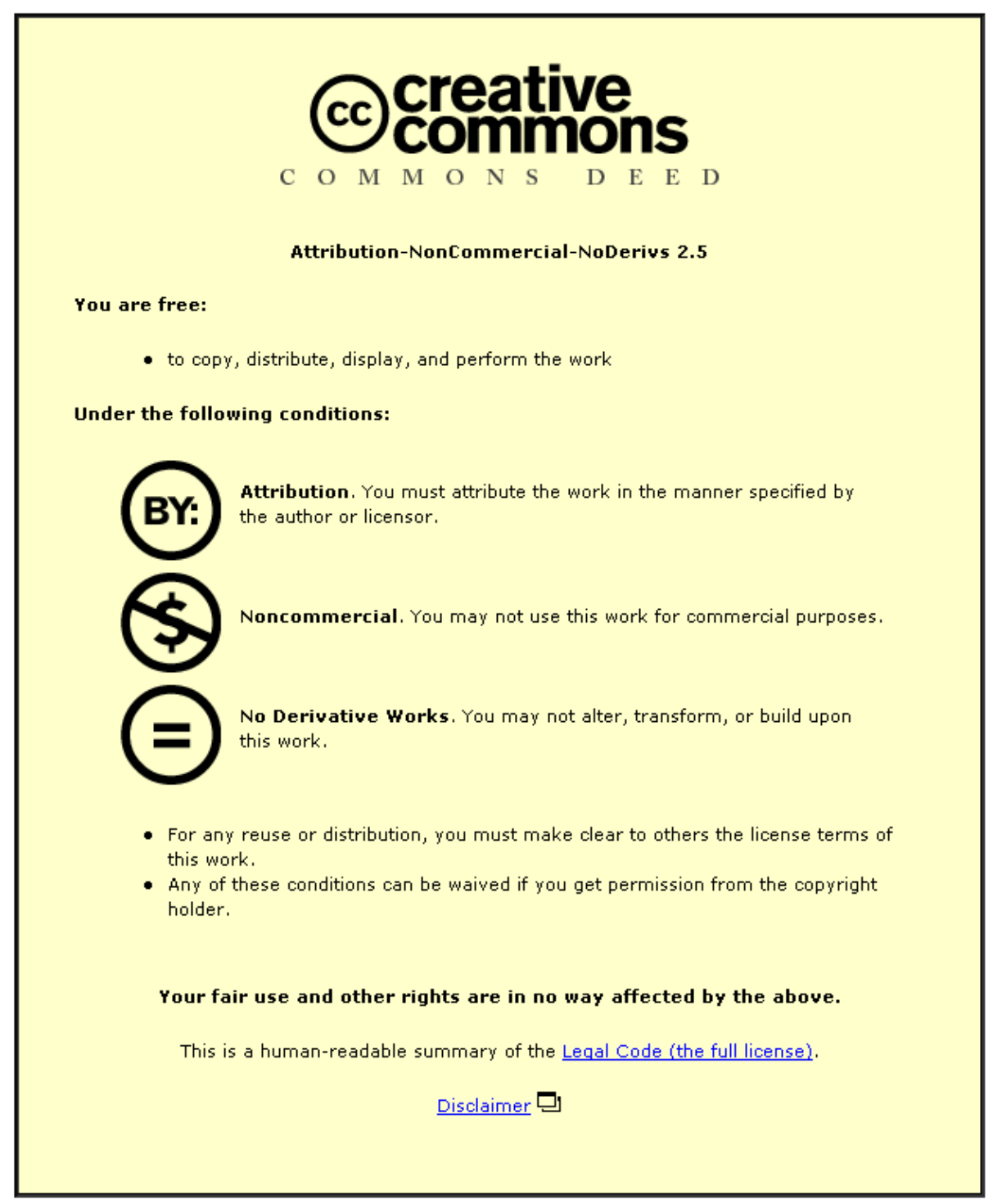

For the full text of this licence, please go to: http://creativecommons.org/licenses/by-nc-nd/2.5/ 


\section{Conductive Lithographic Film Fabricated Resistive Strain Gauges}

\author{
Gareth I. Hay* \\ gareth.hay@brunel.ac.uk \\ Darren Southee* \\ darren.southee@brunel.ac.uk
}

\author{
Peter S. A. Evans* \\ peter.evans@brunel.ac.uk \\ George Simpson* \\ george.simpson@brunel.ac.uk
}

\author{
David J. Harrison* \\ david.harrison@brunel.ac.uk \\ Paul M. Harrey \\ paulh@rotronic.co.uk
}

*Department of Design, Brunel University, Egham, Surrey, UK

- Rotronic Instruments (UK) Ltd, Crawley, West Sussex, UK

\begin{abstract}
This paper reports progress in sensor fabrication by the Conductive Lithographic Film (CLF) printing process. Work describing strain sensitive structures manufactured using a modified printing process and conductive inks are addressed. The performance of a 'single ink' strain sensitive structure when printed on six alternative polymer substrates (GlossArt, PolyArt, Teslin, Mylar C, Mylar and Kapton) is analysed. Though not intending to compete with conventional gauges in high tolerance measurement, the structures exhibit properties that indicate suitability for novel applications.
\end{abstract}

Keywords: Printed strain gauges, Printed sensors, Offset lithography, CLF

\section{INTRODUCTION}

Resistive strain gauges are a popular and reliable method for determining localised strain in manufacturing and engineering industries. Average unit strain, capable of being determined using strain gauges, can be summarised as the "total deformation of a body in a given direction divided by the original length in that direction"[1]. As such, the operation of resistive strain gauges relies on the change in resistance of a conductor when a load is applied.

Common commercially available strain gauge structures are manufactured using conventional photo-resist and acid etching processes. The disadvantages associated with these techniques are generation of toxic effluents and the slow speed of production.

\section{CLF Process}

The Conductive Lithographic Film (CLF) process was originally developed as an alternative for etched resinlaminate circuit boards. The technique utilises standard offset lithographic printing technology used in the mass production of books and magazines. The CLF process possesses a number of key advantages over more traditional forms of electronic circuit board fabrication [2]:

- High production speed $(6,000-10,000$ impressions/hour)
- Good resolution of image (80 - 100 micron track with 60 micron gap easily achievable)

- Low cost (low ink volume determining that substrate material proves the largest expense)

- Ability to produce flexible electronic circuits and systems

- Reduced environmental impact (less energy, reduction in material use, easier disposal, toxic heavy metals (e.g. lead) eliminated)

Electrically conductive ink films can also be printed on a wide range of flexible substrates including paper and polymer films, and which possess similar properties to copper tracking on conventional resin-laminate circuit boards.

A range of passive components and sensor structures have been manufactured by the CLF process by designing structures that exploit the electrical properties of the printed ink films [3],[4]. CLF conductors printed on flexible polymer materials have been found to exhibit a change in their electrical resistance when deformed, lending themselves to resistive strain gauge applications. The aim of this research is to identify the best substrate for printed strain structures and to understand in more detail the electrical and mechanical properties of the ink when deposited on different substrates. Attention is paid to changes in resistance due to strain, relative humidity and temperature.

\section{EXPERIMENTAL METHODS}

Early strain sensitive structures proved to be acceptably sensitive when deformed. However, a large degree of drift was noticed during repeated cycling. Mechanical settling of the ink or changes in ambient temperature or relative humidity was thought to be the cause of such drift. In response, a series of experiments have been undertaken to identify the amount of drift cause by these factors.

\section{Fabrication of Structures}

Strain sensitive structures, Fig. 1, were formed by lithographically printing silver loaded conductive ink onto substrates, using the practice of over printing three times to 
ensure an even, approximately 3 - micron, ink film throughout. The apparatus used for this operation was a sheet-fed lithographic printing press, model Heidelberg GTO46. A novel silver loaded conductive ink developed by Brunel University was used in printing trials. The ink constitutes $80 \%$ silver particulate by weight (1 micron mean size) and achieves a sheet resistance of approximately $0.15 \Omega / \square$.

After printing, samples were allowed to dry in air for 7 days before constantan contact wires were attached using a commercially available conductive adhesive (Circuitworks CW2400). Once the curing time for the adhesive recommended by the manufacture was completed, samples were oven heated at $70^{\circ} \mathrm{c}$ for 15 minutes to evaporate any remaining solvent in the ink films.

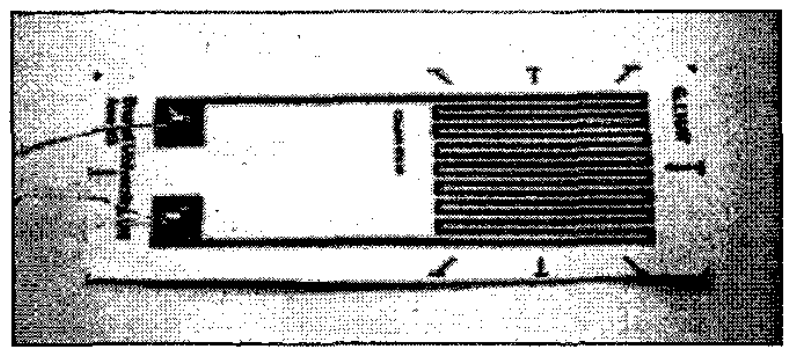

Figure 1. Printed Strain Sensitive Structure

Resistance - Strain Measurements

Aluminium beams $(6.5 \mathrm{~mm} \times 34.5 \mathrm{~mm} \times 600 \mathrm{~mm})$ were employed for the resistance - strain measurements. Beams were prepared before the introduction of samples by following standard strain gauge application procedures.

- Application area treated using Emery cloth and Ammonia water

- Residue removed using Phosphoric acid applied with cotton

- Area finally neutralised using Ammonia water

The substrate surface to be adhered to the beam was coated with an adhesion catalyst $(200$ Catalyst $-C)$, which was allowed to dry before cyanoacrylate adhesive ( $\mathrm{M}$ - Bond 200) was used to form a bond, taking care to eliminate the presence of air pockets between substrate and beam. The assembly was allowed to cure for an hour before soldering lead wires to the contact wires. The nominal resistance of printed sensor structures varied depending on the substrate material, and varied from $60 \Omega-250 \Omega$.

A four-point deflection jig, Fig. 2, was employed to ensure uniform beam deflection during trials. Each structure was exposed to four repetitive straining and relaxing cycles from $0-2000$ micro strain $(\mu \varepsilon)$ while resistance measurements were recorded at $100 \mu \varepsilon$ intervals using a Fluke 189 True RMS Multimeter.

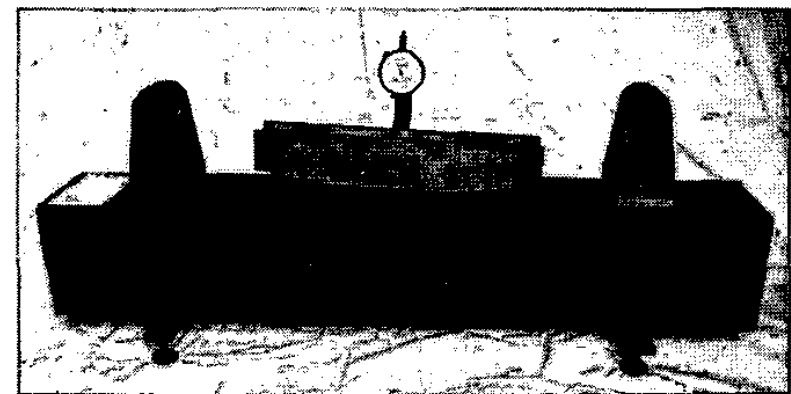

Figure 2. Four - point deflection jig

Temperature-Relative Humidity-Resistance Measurements Temperature - Resistance and Relative Humidity Resistance tests were conducted in order to determine how the resistance of ink films varied when exposed to extremes of temperature and relative humidity. Single track ink film structures printed and prepared in the same manner as the strain sensitive structures were employed, and as with the strain structures initial resistance varied depending on the substrate, from approximately $15 \Omega-100 \Omega$.

For the purpose of humidity testing, a humidity chamber / generator (Digilog Instruments, Vaportron $\mathrm{H}-100 \mathrm{~L}$ ) was adjusted to maintain a constant $23^{\circ} \mathrm{c}$, while relative humidity was cycled in $5 \% \mathrm{RH}$ steps from $20 \% \mathrm{RH}-$ $70 \% \mathrm{RH}$ over three cycles. Resistance measurements were recorded at these intervals using the Fluke multimeter. Temperature testing followed a similar trend; relative humidity was maintained at a constant $40 \% \mathrm{RH}$ while temperature was incremented and decremented from $15^{\circ} \mathrm{c}$ to $40^{\circ} \mathrm{c}$ in $5^{\circ} \mathrm{c}$ steps over three repetitive cycles. Again, resistance measurements were recorded at every interval.

\section{RESULTS}

Relative Humidity - Resistance

Figure 3, details typical responses gained from samples printed on each of the six different substrates while undergoing relative humidity - resistance testing.

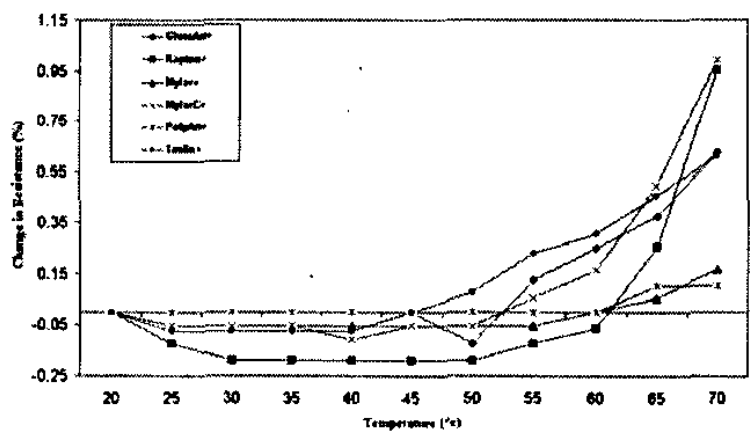

Figure 3. Typical Relative Humidity - Resistance plot for each substrate 
Changes to resistance due to relative humidity (RH) are non-linear, and become significant at humidities above $50 \% \mathrm{RH}$. The greatest change in resistance due to humidity occurred with structures printed on Mylar C and amounted to approximately $0.95 \%$ of the initial resistance.

Table 1. \% Change in Resistance at $50 \%$ RH and 70\%RH

\begin{tabular}{|c|c|c|}
\hline Substrate & $\% \Delta \mathrm{R}$ at $50 \% \mathrm{RH}$ & $\% \Delta \mathrm{R}$ at $70 \% \mathrm{RH}$ \\
\hline GlossArt & -0.125 & 0.626 \\
\hline PolyArt & 0 & 0.104 \\
\hline Teslin & 0.076 & 0.612 \\
\hline Mylar C & -0.055 & 0.991 \\
\hline Mylar & -0.056 & 0.168 \\
\hline Kapton & -0.191 & 0.953 \\
\hline
\end{tabular}

Refering to Table 1, it is apparent that structures printed on Kapton experienced the greatest change in resistance due to humidity at $50 \% \mathrm{RH}(-0.191 \%$ of initial resistance $)$. The resistance of PolyArt remains stable under these conditions. Between the range of $50 \% \mathrm{RH}-70 \% \mathrm{RH}$ the resistance of all substrates increase dramatically. Mylar $\mathrm{C}$ experienced the greatest increase while PolyArt remained the least affected substrate.

\section{Temperature - Resistance}

Typical results gained during temperature - resistance tests for structures printed on each of the six substrates are illustrated in Figure 4 . The trend of increasing resistance with temperature can be observed.

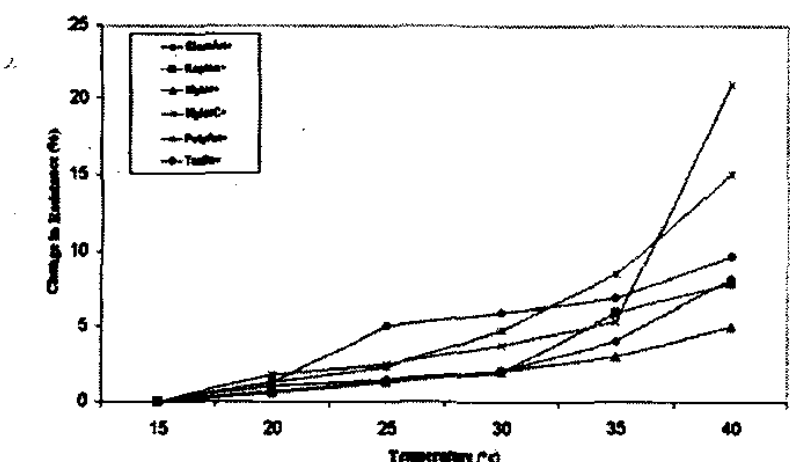

Figure 4. Typical Temperature-Resistance plot for each substrate

As with data gained from relative humidity - resistance tests, results show a large degree on non-linearity, this becomes more noticeable through the range of $30^{\circ} \mathrm{c}-40^{\circ} \mathrm{c}$ where resistance increases at an accelerated rate. Table 2 details in a numerical form the large acceleration in resistance increase over this range.

Kapton is the least affected substrate during testing while PolyArt is the most affected substrate over the range $0^{\circ} \mathrm{c}$ $30^{\circ} \mathrm{c}$. Above $35^{\circ} \mathrm{c}$ the resistance of Mylar $\mathrm{C}$ in particular increases rapidly, this trend was noticed during all temperature tests using this substrate.

Due to the extreme non-linearity experienced at high temperatures the Temperature Coefficient of Resistance (TCR) for each substrate was determined, using the equation below, by considering results in the range $15^{\circ} \mathrm{c}-$ $35^{\circ} \mathrm{c}$ and are detailed in Table 2.

$$
\alpha=\left(\frac{1}{R}\right) *\left(\frac{\Delta R}{\Delta T}\right)
$$

In the TCR equation, $\alpha$ represents the temperature coefficient of resistance, $R$ represents the nominal resistance and $\Delta R$ and $\Delta T$ represent the change in resistance and the change in temperature respectively.

It is possible to determine that over this range PolyArt was affected the most due to changes in ambient temperature and Mylar was affected the least. It must be noted that all samples were printed at the same time with the same batch of ink and therefore any changes in resistance due to either relative humidity or temperature reflects the compatibility of ink to substrate. Substrates such as Teslin, PolyArt and GlossArt have been developed for graphical applications in commercial printing. Substrates such as Mylar, Mylar C and Kapton are engineering materials specified for electronic applications.

Table 2. \% Change in Resistance at $30^{\circ} \mathrm{c}$ and $40^{\circ} \mathrm{C}$. Also Temperature Coefficient of Resistance

\begin{tabular}{|c|c|c|c|}
\hline Substrate & $\% \Delta \mathrm{R}-30^{\circ} \mathrm{c}$ & $\% \Delta \mathrm{R}-40^{\circ} \mathrm{c}$ & $\begin{array}{c}\mathrm{TCR} \\
\left({ }^{*} 10^{-3} /{ }^{\circ} \mathrm{c}\right)\end{array}$ \\
\hline GlossArt & 2.058 & 8.162 & 2.023 \\
\hline PolyArt & 4.715 & 15.054 & 4.218 \\
\hline Teslin & 5.879 & 9.671 & 3.4134 \\
\hline Mylar C & 3.759 & 20.930 & 2.6441 \\
\hline Mylar & 2.065 & 5.004 & 1.5091 \\
\hline Kapton & 1.954 & 7.815 & 2.9307 \\
\hline
\end{tabular}

\section{Strain - Resistance}

The strain - resistance response of the structures are depicted in Figures 5 and 6 . These illustrations chart fractional change in resistance against micro strain for the various printed strain sensitive structures evaluated.

The characteristics of sensors printed on Teslin are plotted as a separate graph as the sensitivity achieved with these samples is significantly greater than those achieved with other substrate materials. 


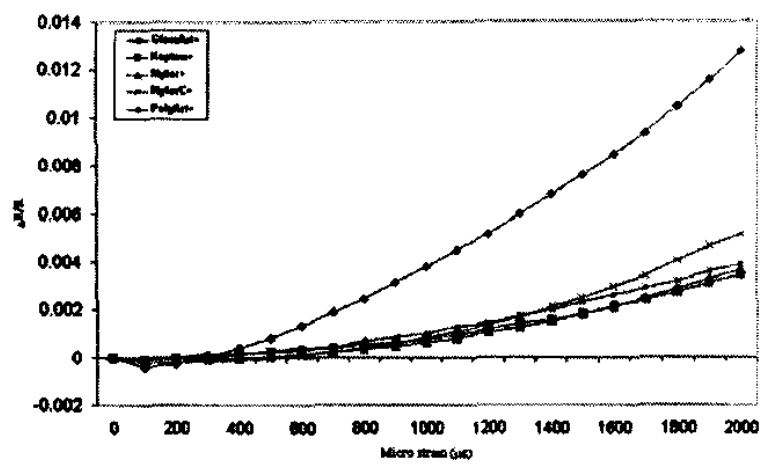

Figure 5. Typical Strain - Resistance plot for each substrate

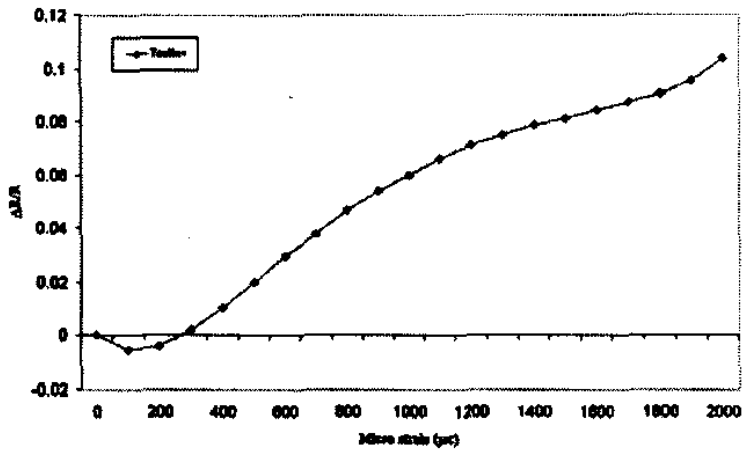

Figure 6. Typical Strain - Resistance plot for Teslin

It is clear from the illustrations that all samples exhibit nonlinear characteristics. In order to compare the degree of linearity between samples, Pearson's Product Moment Correlation Coefficients [PPMCC] were generated from the data and are depicted in Table 3. From these values it was determined that the response of Teslin sensors demonstrated the greatest degree of linearity while Mylar C sensors demonstrated the worst linearity. Measurements of linearity for all printed structures are comparable to other printed thick film sensors [5].

The data displayed in Figures 5 and 6 was used to generate gauge factor values for each printed structure. The gauge factor (GF) is derived to numerically represent the sensitivity of a structure by considering the change in resistance, nominal resistance and applied strain.

$$
G F=\frac{\Delta R / R}{\varepsilon}
$$

Gauge factor values were derived using the equation above, where $\Delta R / R$ represents the fractional change in resistance and $\varepsilon$ represents the applied strain, and are listed in Table 3.
Table 3. Gauge Factor and Pearson's Product Moment Correlation Coefficient for each substrate

\begin{tabular}{|c|c|c|}
\hline Substrate & Gauge Factor & PPMCC \\
\hline GlossArt & 6.388 & 0.961 \\
\hline PolyArt & 1.947 & 0.942 \\
\hline Teslin & 51.851 & 0.968 \\
\hline Mylar C & 2.582 & 0.885 \\
\hline Mylar & 1.832 & 0.890 \\
\hline Kapton & 1.711 & 0.928 \\
\hline
\end{tabular}

Gauge Factor (GF) values confirm the trends represented in Figures 5 and 6, indicating that structures printed on Teslin are the most sensitive, achieving a GF of approximately 52 . Structures printed on Kapton are least sensitive, achieving a GF of approximately 1.7. Structures printed on the remaining substrates possess gauge factors between 1.8 and 6.3 .

Figures 7 and 8 represent typical reproducibility plots for GlossArt and Kapton respectively.

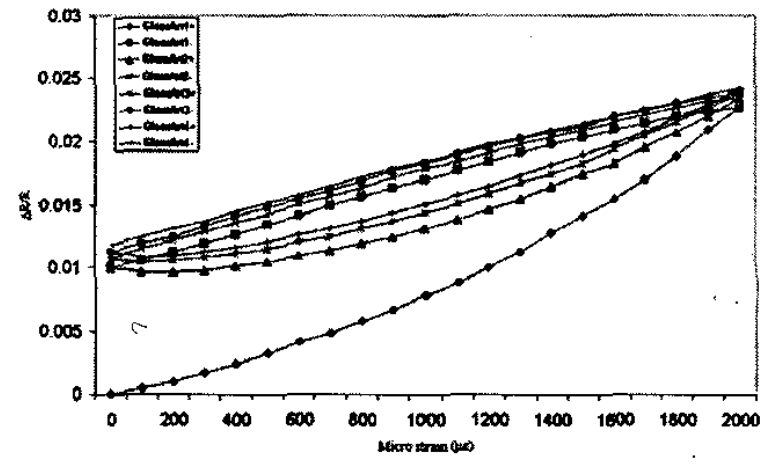

Figure 7. Typical repeatability plot for GlossArt

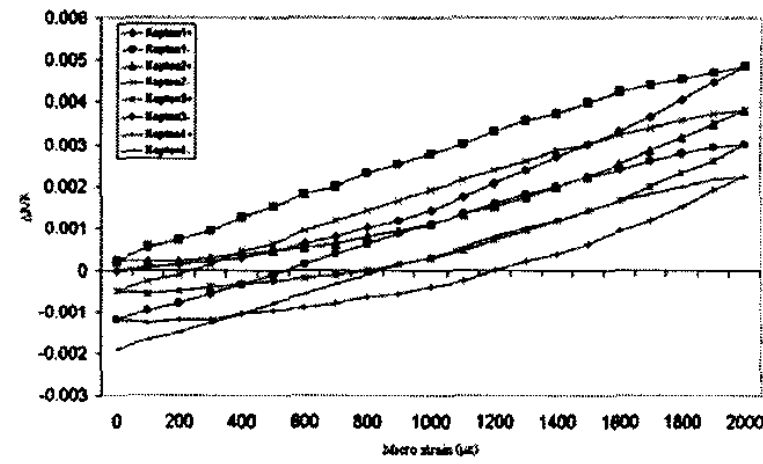

Figure 8. Typical repeatability plot for Kapton

Readings of fractional change in resistance taken from all structures are consistent over repeated loading - unloading cycles. Figures 7 and 8 depict the reproducibility of all substrate responses to strain despite possessing different characteristics. Figure 7 indicates a large degree of 
hysteresis on the first cycle while subsequent cycles incur a small amount of drift. This is comparable to the results obtained from Mylar $\mathrm{C}$ and PolyArt samples. Figure 8 indicates continuous cyclical hysteresis but also significant drift between cycles. These trends are also apparent in sensors printed on Teslin and Mylar substrates.

\section{CONCLUSION}

A range of strain sensitive structures fabricated by the Conductive Lithographic Film (CLF) printing process have been evaluated. The results demonstrate that these structures exhibit comparable strain-resistance responses to similar sensors fabricated by conventional screen-printing techniques[5].

The response of these structures to changes in temperature and relative humidity have also been quantified. Changes in sensor resistance due to changes in relative humidity prove to be negligible when compared to the changes in resistance caused by changes in ambient temperature.

During strain - resistance measurements, local monitoring of ambient temperature and relative humidity was performed to ensure that these variables did not significantly affect the data being gathered.

We conclude that hysteresis observed in characterising these printed strain sensors is attributed to mechanical changes in the conductive lithographic ink films under strain, rather than changes in relative humidity or ambient temperature.

\section{ACKNOWLEDGMENT}

The authors acknowledge the assistance of staff at Rotronic Instruments (UK) Ltd, Crawley, for their assistance in gathering data in support of this work.

\section{REFERENCES}

[1] C. C. Perry, H. R. Lissner

"The Strain Gage Primer". Second edition

1962 McGraw - Hill Book Company

ISBN 07-049461-4

[2] B. J. Ramsey, P. S. A. Evans \& D. J. Harrison "A Novel Circuit Fabrication Technique Using Offset Lithography". Journal of Electronics Manufacture, Vol. No.7, No.1, 1997.

[3] N. S. Leyland, J. R. G. Evans, D. J. Harrison "Lithographic Printing of Force - Sensitive Resistors". Journal of Material Science: Materials in Electronics 13 (2002). pp $387-390$.

[4] P.R. Shepherd, C. Taylor, P.S.A. Evans \& D. J. Harrison

"Measurement and Modelling of MIC Components Using Conductive Lithographic Films". 31st European

Microwave Conference - London 2001.

[5] K. I. Arshak, A. K. Ray, C. A. Hogarth, D. G Collins, F. Ansari

"An analysis of polymeric thick-film resistors as pressure sensors". Sensors and Actuators A 49 (1995). pp 41 - 45 